ANNALES

POLONICI MATHEMATICI

$93.2(2008)$

\title{
Coefficient inequalities for concave and meromorphically starlike univalent functions
}

\author{
by B. Bhowmik and S. Ponnusamy (Chennai)
}

\begin{abstract}
Let $\mathbb{D}$ denote the open unit disk and $f: \mathbb{D} \rightarrow \overline{\mathbb{C}}$ be meromorphic and univalent in $\mathbb{D}$ with a simple pole at $p \in(0,1)$ and satisfying the standard normalization $f(0)=f^{\prime}(0)-1=0$. Also, assume that $f$ has the expansion

$$
f(z)=\sum_{n=-1}^{\infty} a_{n}(z-p)^{n}, \quad|z-p|<1-p,
$$

and maps $\mathbb{D}$ onto a domain whose complement with respect to $\overline{\mathbb{C}}$ is a convex set (starlike set with respect to a point $w_{0} \in \mathbb{C}, w_{0} \neq 0$ resp.). We call such functions concave (meromorphically starlike resp.) univalent functions and denote this class by $\operatorname{Co}(p)\left(\Sigma^{\mathrm{s}}\left(p, w_{0}\right)\right.$ resp.). We prove some coefficient estimates for functions in these classes; the sharpness of these estimates is also established.
\end{abstract}

1. Introduction. One of the most interesting questions in the theory of univalent functions is to find the region of variability of the $n$th Taylor (Laurent resp.) coefficient for functions $f$ that are analytic (meromorphic resp.) and univalent in the unit disk $\mathbb{D}=\{z:|z|<1\}$. The leading example is the Bieberbach conjecture settled by de Branges in 1985 for the class $\mathcal{S}$ of normalized analytic univalent functions $f$ in $\mathbb{D}$ although corresponding results for important subclasses of $\mathcal{S}$ are relatively easy and were settled positively much earlier.

In this paper, we consider the family $\mathrm{Co}(p)$ of functions $f: \mathbb{D} \rightarrow \overline{\mathbb{C}}$ that satisfy the following conditions:

(i) $f$ is meromorphic in $\mathbb{D}$ and has a simple pole at the point $p \in(0,1)$ with the standard normalization $f(0)=f^{\prime}(0)-1=0$.

(ii) $f$ maps $\mathbb{D}$ conformally onto a set whose complement with respect to $\overline{\mathbb{C}}$ is convex.

2000 Mathematics Subject Classification: Primary 30C45.

Key words and phrases: Laurent coefficients, meromorphic univalent functions, concave functions, starlike functions, convex set. 
Each $f \in \operatorname{Co}(p)$ has the power series expansion of the form

$$
f(z)=z+\sum_{n=2}^{\infty} A_{n}(f) z^{n}, \quad|z|<p .
$$

For our investigation, we consider the Laurent expansion of $f \in \operatorname{Co}(p)$ about the pole $z=p$ :

$$
f(z)=\sum_{n=-1}^{\infty} a_{n}(z-p)^{n}, \quad z \in \Delta_{p},
$$

where $\Delta_{p}=\{z \in \mathbb{C}:|z-p|<1-p\}$. Motivated by the works of Pfaltzgraff and Pinchuk [8], Miller [7], and Livingston [6], the class $\operatorname{Co}(p)$ has been investigated recently in $[4,1,2,3,10]$. A necessary and sufficient condition for a function $f$ to be in $\operatorname{Co}(p)([6])$ is that $\operatorname{Re} \phi(z, f)>0$ for all $z \in \mathbb{D}$, where

$$
\phi(z, f)=-\left(1+p^{2}\right)+2 p z-\frac{(z-p)(1-p z) f^{\prime \prime}(z)}{f^{\prime}(z)}, \quad z \in \mathbb{D} .
$$

Livingston [6] determined some estimates of the real part of $A_{n}(f)$ for $n=2,3$ when $f \in \operatorname{Co}(p)$ has the expansion (1.1). In the same article he conjectured an estimate for the real part of the general coefficient $A_{n}(f)$ $(n \geq 2)$ for $f \in \mathrm{Co}(p)$. After a long gap of ten years, positive developments have occurred in this line of work. For example, the recent work of Avkhadiev and Wirths [4] settles the conjecture of Avkhadiev, Pommerenke and Wirths [1] which, in particular, provides a proof of the Livingston conjecture. For ease of reference, we now recall it here.

Theorem A ([4]). Let $n \geq 2$ and $p \in(0,1)$. For each $f \in \mathrm{Co}(p)$ with the expansion (1.1) the inequality

$$
\left|A_{n}(f)-\frac{1-p^{2 n+2}}{p^{n-1}\left(1-p^{4}\right)}\right| \leq \frac{p^{2}\left(1-p^{2 n-2}\right)}{p^{n-1}\left(1-p^{4}\right)}
$$

is valid. Equality is attained in (1.3) if and only if $f$ is one of the functions $f_{\theta}, \theta \in[0,2 \pi)$, where

$$
f_{\theta}(z)=\frac{z-\frac{p}{1+p^{2}}\left(1+e^{i \theta}\right) z^{2}}{(1-z / p)(1-z p)} .
$$

For each complex number in the disk described in (1.3) there exists a function $f \in \mathrm{Co}(p)$ such that this number occurs as the nth Taylor coefficient of $f$.

Interestingly, Wirths [10] established the following representation formula for functions in $\mathrm{Co}(p)$.

Theorem B ([10]). For each $f \in \mathrm{Co}(p)$, there exists a function $\omega$ holomorphic in $\mathbb{D}$ such that $\omega(\mathbb{D}) \subset \overline{\mathbb{D}}$ and 


$$
f(z)=\frac{z-\frac{p}{1+p^{2}}(1+\omega(z)) z^{2}}{(1-z / p)(1-z p)}, \quad z \in \mathbb{D} .
$$

The above representation formula has been used by the authors in [5] to obtain some other kind of coefficient estimates for functions in the class $\mathrm{Co}(p)$ with the Laurent expansion of the form (1.2).

In the present article, we first obtain certain coefficient estimates for functions in $\operatorname{Co}(p)$ with the same expansion of the form (1.2). Next we discuss a related class of meromorphically starlike functions, namely, the class $\Sigma^{\mathrm{s}}\left(p, w_{0}\right)$, and obtain a simple and easily applicable representation formula for this class. Using this formula, we also obtain some sharp coefficient estimates for functions in this class. As a consequence, we rectify a mistake in [6, Theorem 9].

Now, we state our first result.

Theorem 1.1. Let $p \in(0,(\sqrt{5}-1) / 2]$ and $f \in \operatorname{Co}(p)$ have the expansion (1.2). Then

$$
\left|p-\left(1-p^{2}\right) \frac{a_{0}}{a_{-1}}\right| \leq \frac{p}{\left|a_{-1}\right|}, \quad \text { i.e. } \quad\left|a_{-1}-\frac{1-p^{2}}{p} a_{0}\right| \leq 1 .
$$

The inequality is sharp.

REMARK. In [10] Wirths has obtained the region of variability for $a_{-1}(f)$, namely, the inequality

$$
\left|a_{-1}+\frac{p^{2}}{1-p^{4}}\right| \leq \frac{p^{4}}{1-p^{4}} \quad \text { for } 0<p<1 .
$$

In [5], the domain of variability of $a_{0}(f)$ is determined by the inequality

$$
\left|\frac{\left(1-p^{2}\right) a_{0}}{p}+\frac{1-p^{2}+p^{4}}{1-p^{4}}\right| \leq \frac{p^{2}\left(2-p^{2}\right)}{1-p^{4}} \quad \text { for } p \in(0, \sqrt{3}-1] .
$$

Equality in each of the above two inequalities is attained if and only if $f$ is one of the functions given in (1.4).

The next result presents sharp coefficient estimates for all $n \geq 3$ if $f \in$ $\mathrm{Co}(p)$ has the expansion (1.2).

THEOREM 1.2. If $f \in \mathrm{Co}(p)$ with $p \in(0,1)$ and has the expansion (1.2), then for $n \geq 3$ we have

$$
\begin{aligned}
& \left|a_{n-2}-\frac{\left(1-p^{2}\right) a_{n-1}}{p}\right| \\
& \quad \leq \frac{p}{\left(1-p^{4}\right)(1-p)^{n-1}}\left[1-\left(\frac{1-p^{4}}{p^{4}}\right)^{2}\left|a_{-1}+\frac{p^{2}}{1-p^{4}}\right|^{2}\right] .
\end{aligned}
$$

Equality holds for the functions $f_{\theta}(0 \leq \theta \leq 2 \pi)$ of the form (1.4). 


\section{Proofs of Theorems 1.1 and 1.2}

(2.1) Proof of Theorem 1.1. Let $f \in \mathrm{Co}(p)$. Then, by Theorem B, there exists a function $\omega$ holomorphic in $\mathbb{D}$ with $\omega(\mathbb{D}) \subset \overline{\mathbb{D}}$ satisfying the representation formula (1.5).

Now, let $f \in \operatorname{Co}(p)$ have the Laurent expansion (1.2) and let $\omega$ have the Taylor expansion

$$
\omega(z)=\sum_{n=0}^{\infty} c_{n}(z-p)^{n}, \quad z \in \Delta_{p} .
$$

Using these two expansions, the series formulation of (1.5) takes the form

$$
\begin{aligned}
& (z-p)\left((z-p)-\frac{1-p^{2}}{p}\right) \sum_{n=-1}^{\infty} a_{n}(z-p)^{n} \\
= & p+(z-p)-\frac{p}{1+p^{2}}\left(1+\sum_{n=0}^{\infty} c_{n}(z-p)^{n}\right)\left((z-p)^{2}+2 p(z-p)+p^{2}\right) .
\end{aligned}
$$

Comparing the coefficients of $z-p$ on both sides of (2.3), we see that

$$
a_{-1}-\frac{1-p^{2}}{p} a_{0}=\frac{1-p^{2}}{1+p^{2}}-\frac{p^{2}}{1+p^{2}}\left(2 c_{0}+p c_{1}\right) .
$$

Using the classical Schwarz-Pick lemma, it follows that

$$
\left|\omega^{\prime}(p)\right| \leq \frac{1-|\omega(p)|^{2}}{1-p^{2}}, \quad \text { i.e. } \quad\left|c_{1}\right| \leq \frac{1-\left|c_{0}\right|^{2}}{1-p^{2}} .
$$

In view of this observation, we have the estimate

$$
\left|2 c_{0}+p c_{1}\right| \leq \frac{p\left(1-\left|c_{0}\right|^{2}\right)+2\left(1-p^{2}\right)\left|c_{0}\right|}{1-p^{2}} .
$$

For convenience, we set $x=\left|c_{0}\right|$ and consider

$$
R_{p}(x)=p\left(1-x^{2}\right)+2\left(1-p^{2}\right) x .
$$

We see that $R_{p}(x)$ attains a local maximum at $x_{m}=\left(1-p^{2}\right) / p$. Since $x_{m} \geq 1$ for $p \in(0,(\sqrt{5}-1) / 2]$, we see that

$$
\left|R_{p}(x)\right| \leq R_{p}(1)=2\left(1-p^{2}\right), \quad x \in[0,1], p \in(0,(\sqrt{5}-1) / 2],
$$

and therefore, we have the estimate $\left|2 c_{0}+p c_{1}\right| \leq 2$ for $p$ in that interval. Now using this we get from (2.4) the estimate (1.6). It is a simple exercise to see that equality is attained in (1.6) for the function

$$
f(z)=\frac{-z p}{(z-p)(1-z p)}
$$


(2.5) Proof of Theorem 1.2. Let $f \in \mathrm{Co}(p)$ with the expansion (1.2). Next, following the notation of the proof of Theorem 1.1, we compare the coefficients of $(z-p)^{n}(n \geq 3)$ on both sides of the equation (2.3). This gives

$$
a_{n-2}-\frac{1-p^{2}}{p} a_{n-1}=-\frac{p}{1+p^{2}}\left(c_{n-2}+2 p c_{n-1}+p^{2} c_{n}\right) \quad(n \geq 3) .
$$

Now, for a unimodular bounded analytic function $\omega$ in the unit disk $\mathbb{D}$ having the expansion (2.2) in $\Delta_{p}$, we recall the following result due to Ruscheweyh $[9$, Theorem 2]:

$$
(1-p)^{n}(1+p)\left|c_{n}\right| \leq 1-\left|c_{0}\right|^{2} \quad(n \geq 1)
$$

where equality holds for $\omega(z)=e^{i \theta}, \theta \in[0,2 \pi)$. Using this, we easily obtain

$$
\left|a_{n-2}-\frac{\left(1-p^{2}\right) a_{n-1}}{p}\right| \leq \frac{p\left(1-\left|c_{0}\right|^{2}\right)}{\left(1+p^{2}\right)(1+p)(1-p)^{n}} \quad(n \geq 3) .
$$

Consequently, (1.7) follows since

$$
c_{0}=\frac{1-p^{4}}{p^{4}} a_{-1}+\frac{1}{p^{2}},
$$

by comparing the constant terms on both sides of (2.3). Now, equality holds of (1.7) for the functions $f_{\theta}, \theta \in[0,2 \pi)$, of (1.4), since both sides of the inequality are zero.

3. Meromorphically starlike functions. Let $\Sigma^{\mathrm{s}}\left(p, w_{0}\right)$ denote the class of meromorphic and univalent functions $f$ in $\mathbb{D}$ (with the standard normalization $f(0)=f^{\prime}(0)-1=0$ ) having a simple pole at $p \in(0,1)$ with the expansion (1.2) such that $f$ is starlike with respect to a fixed $w_{0} \in \mathbb{C}, w_{0} \neq 0$ (i.e. $\overline{\mathbb{C}} \backslash f(\mathbb{D})$ is a starlike set with respect to $w_{0}$ ). A wellknown fact [6] is that $f \in \Sigma^{\mathrm{S}}\left(p, w_{0}\right)$ if and only if $\operatorname{Re} \psi(z, f)>0$ for all $z \in \mathbb{D}$, where

$$
\psi(z, f)=\frac{-(z-p)(1-p z) f^{\prime}(z)}{f(z)-w_{0}}, \quad z \in \mathbb{D} .
$$

We now prove a useful representation formula for functions in the class $\Sigma^{\mathrm{S}}\left(p, w_{0}\right)$.

Theorem 3.1. For $0<p<1$, let $f \in \Sigma^{\mathrm{S}}\left(p, w_{0}\right)$. Then there exists a function $\omega$ holomorphic in $\mathbb{D}$ such that $\omega(\mathbb{D}) \subset \overline{\mathbb{D}}$,

$$
\omega(0)=-\frac{1}{2}\left(\frac{1}{w_{0}}+p+\frac{1}{p}\right)
$$

and

$$
f(z)=w_{0}+\frac{p w_{0}(1+z \omega(z))^{2}}{(z-p)(1-z p)}, \quad z \in \mathbb{D}
$$


Proof. The proof is a direct consequence of [12, Corollary 2] where the notation $\sigma^{*}\left(p, w_{0}\right)$ is used in place of $\Sigma^{\mathrm{S}}\left(p, w_{0}\right)$. By that corollary, if $f \in$ $\Sigma^{\mathrm{S}}\left(p, w_{0}\right)$, then

Now writing

$$
\left|\left\{\frac{f(z)-w_{0}}{p w_{0}}(z-p)(1-p z)\right\}^{1 / 2}-1\right| \leq|z|, \quad z \in \mathbb{D} .
$$

$$
\omega(z)=\frac{1}{z}\left\{\frac{f(z)-w_{0}}{p w_{0}}(z-p)(1-p z)\right\}^{1 / 2}-\frac{1}{z}
$$

and simplifying the above expression for $f$ we get the desired representation formula for functions in the class $\Sigma^{\mathrm{s}}\left(p, w_{0}\right)$. Here we note that $\omega$ is holomorphic in $\mathbb{D}$ and $|\omega(z)| \leq 1$. Also since $f^{\prime}(0)=1$ we get $\omega(0)=$ $-\frac{1}{2}\left(1 / w_{0}+p+1 / p\right)$.

As a consequence of Theorem 3.1, we have the following result which has been proved in [11] by using a different method.

Corollary 3.1. For $0<p<1$, let $f \in \Sigma^{\mathrm{S}}\left(p, w_{0}\right)$. Then

$$
\left|w_{0}+\frac{p\left(1+p^{2}\right)}{\left(1-p^{2}\right)^{2}}\right| \leq \frac{2 p^{2}}{\left(1-p^{2}\right)^{2}} .
$$

In particular,

$$
\frac{p}{(1+p)^{2}} \leq\left|w_{0}\right| \leq \frac{p}{(1-p)^{2}}
$$

Proof. As $|\omega(0)| \leq 1$ and $\omega(0)=-\frac{1}{2}\left(1 / w_{0}+p+1 / p\right)$, it follows that

$$
\left|\frac{1}{w_{0}}+\frac{1+p^{2}}{p}\right| \leq 2
$$

which is easily seen to be equivalent to the inequality (3.3). The other inequality is a simple consequence of (3.3). Then

Theorem 3.2. Let $f \in \Sigma^{\mathrm{S}}\left(p, w_{0}\right)$ have the Laurent expansion (1.2).

$$
\begin{aligned}
& \left|a_{-1}-\frac{p w_{0}}{1-p^{2}}\right| \\
& \quad \leq \frac{p\left|w_{0}\right|}{1-p^{2}} \frac{\left|p / w_{0}+p^{2}+1\right|+2 p^{2}}{2+\left|p / w_{0}+p^{2}+1\right|}\left(\frac{\left|p / w_{0}+p^{2}+1\right|+2 p^{2}}{2+\left|p / w_{0}+p^{2}+1\right|}+2\right)
\end{aligned}
$$

for $p \in(0,1)$,

$$
\left|a_{0}-\frac{1-p^{2}+p^{4}}{\left(1-p^{2}\right)^{2}} w_{0}\right| \leq \frac{p\left(2+2 p-p^{3}\right)}{\left(1-p^{2}\right)^{2}}\left|w_{0}\right|, \quad p \in(0,(\sqrt{5}-1) / 2] .
$$

Both inequalities are sharp for

$$
f(z)=\frac{-z p}{(z-p)(1-p z)}=w_{0}+\frac{p w_{0}}{(z-p)(1-p z)}(1-z)^{2} \in \Sigma^{s}\left(p, w_{0}\right)
$$

where $w_{0}=-p /(1+p)^{2}$. 
Proof. Consider the Taylor expansion for $\omega$ :

$$
\omega(z)=\sum_{n=0}^{\infty} c_{n}(z-p)^{n}, \quad z \in \Delta_{p} .
$$

Now substituting (1.2) and (2.2) in the representation formula (3.2) we get the following series formulation of (3.2) valid in $\Delta_{p}$ :

$$
\begin{aligned}
& \sum_{n=-1}^{\infty} a_{n}(z-p)^{n}-w_{0} \\
& =\frac{p w_{0}}{1-p^{2}} \sum_{n \geq 0}\left(\frac{p}{1-p^{2}}\right)^{n}(z-p)^{n-1} \\
& \quad \times\left[1+\left\{(z-p)^{2}+p^{2}+2 p(z-p)\right\} \sum_{n \geq 0}\left(\sum_{k=0}^{n} c_{k} c_{n-k}\right)(z-p)^{n}\right. \\
& \left.\quad+\{2 p+2(z-p)\} \sum_{n \geq 0} c_{n}(z-p)^{n}\right] .
\end{aligned}
$$

In deriving the above expression, we make use of the relations

$$
\begin{aligned}
(z-p)(1-p z)= & \left(1-p^{2}\right)(z-p)\left(1-\frac{p}{1-p^{2}}(z-p)\right) \\
(1+z \omega(z))^{2}= & 1+2(z-p+p) w(z) \\
& +\left((z-p)^{2}+2 p(z-p)+p^{2}\right) w(z) w(z) .
\end{aligned}
$$

Now, we proceed to prove (i). Comparing the coefficients of $1 /(z-p)$ on both sides of (3.4) we get

$$
a_{-1}=\frac{p w_{0}}{1-p^{2}}\left[1+p^{2} c_{0}^{2}+2 p c_{0}\right] .
$$

The Schwarz-Pick lemma applied to $\omega$ shows that

$$
\left|c_{0}\right|=|\omega(p)| \leq \frac{|\omega(0)|+p}{1+p|\omega(0)|}
$$

where

$$
|\omega(0)|=\frac{1}{2}\left|\frac{1}{w_{0}}+p+\frac{1}{p}\right| .
$$

Using this, we get the desired estimate for $a_{-1}$. It is also easy to check that (i) is sharp for the function given in the statement.

(ii) Comparing the constant terms on both sides of (3.4) we get

$$
a_{0}-w_{0}=\frac{p^{2} w_{0}}{\left(1-p^{2}\right)^{2}}\left(1+p^{2} c_{0}^{2}+2 p c_{0}\right)+\frac{2 p w_{0}}{1-p^{2}}\left(p^{2} c_{0} c_{1}+p^{2} c_{0}^{2}+p c_{1}+c_{0}\right),
$$


or equivalently, $a_{0}-\frac{1-p^{2}+p^{4}}{\left(1-p^{2}\right)^{2}} w_{0}=\frac{p^{2} w_{0}}{\left(1-p^{2}\right)^{2}}\left(p^{2} c_{0}^{2}+2 p c_{0}\right)+\frac{2 p w_{0}}{1-p^{2}}\left(p^{2} c_{0} c_{1}+p^{2} c_{0}^{2}+p c_{1}+c_{0}\right)$.

Now, we recall the estimates from the Schwarz-Pick lemma:

$$
\left|c_{0}\right| \leq 1, \quad\left|c_{1}\right| \leq \frac{1-\left|c_{0}\right|^{2}}{1-p^{2}}
$$

For convenience, we use the notation $x=\left|c_{0}\right|$. Using the above estimates, it is easy to see that the last equality implies that

$$
\left|a_{0}-\frac{1-p^{2}+p^{4}}{\left(1-p^{2}\right)^{2}} w_{0}\right| \leq \frac{p\left|w_{0}\right|}{\left(1-p^{2}\right)^{2}}\left(2 p+2 x+2 p^{2} x-2 p^{2} x^{3}-p^{3} x^{2}\right) .
$$

Next, we introduce

$$
Q_{p}(x)=2 p+2 x+2 p^{2} x-2 p^{2} x^{3}-p^{3} x^{2}, \quad 0 \leq x \leq 1 .
$$

Then $Q_{p}$ attains a local maximum at

$$
x_{m}=\left(-p^{2}+\sqrt{p^{4}+12\left(1+p^{2}\right)}\right) /(6 p) .
$$

Since $x_{m} \geq 1$ for $p \in(0,(\sqrt{5}-1) / 2]$, we have

$$
\max \left\{Q_{p}(x): x \in[0,1]\right\}=Q_{p}(1)=2+2 p-p^{3} .
$$

This proves inequality (ii), and the sharpness can easily be verified for the function given in the statement.

REMARK. It is a simple exercise to see that

$$
\frac{\left|p / w_{0}+p^{2}+1\right|+2 p^{2}}{2+\left|p / w_{0}+p^{2}+1\right|} \leq p
$$

is equivalent to

$$
\frac{1}{2}\left|\frac{p}{w_{0}}+p^{2}+1\right| \leq p, \quad \text { i.e. } \quad|\omega(0)| \leq 1 .
$$

Thus, (3.5) holds. If we use the inequality (3.5) then inequality (i) of Theorem 3.2 turns out to be

$$
\left|a_{-1}-\frac{p w_{0}}{1-p^{2}}\right| \leq \frac{p^{2}}{1-p^{2}}(p+2)\left|w_{0}\right|, \quad p \in(0,1) .
$$

Applying the triangle inequality and inequality (ii) of Theorem 3.2 we get

$$
\left|a_{-1}\right| \leq \frac{p(1+p)}{1-p}\left|w_{0}\right|, \quad p \in(0,1)
$$

and

$$
\left|a_{0}\right| \leq \frac{1}{(1-p)^{2}}\left|w_{0}\right|, \quad p \in(0,(\sqrt{5}-1) / 2],
$$

respectively. Both the above estimates are sharp for the function stated in Theorem 3.2. 
The estimate (3.6) shows that there was a minor error in one of the results of Livingston, namely Theorem 9 in [6]. Indeed, a counterexample is given by the function

$$
g(z)=\frac{-z p}{(z-p)(1-p z)} \in \Sigma^{\mathrm{S}}\left(p, \frac{-p}{1+p^{2}}\right) .
$$

Here we note that

$$
a_{-1}(g)=\frac{-p^{2}}{1-p^{2}}
$$

does not belong to the disk stated in Theorem 9 of [6]. Moreover, the error actually occurred in $[6$, p. 290] where the inequality in the 6th line needs to be reversed, since $\xi-p \leq 0$. We can now formulate a corrected version of [6, Theorem 9] for future use.

Theorem 3.3. If $f \in \Sigma^{\mathrm{s}}\left(p, w_{0}\right)$ and has the Laurent expansion (1.2), then

$$
\left|a_{-1}\right| \geq \frac{p(1-p)}{1+p}\left|w_{0}\right|
$$

The inequality is sharp for the function

$$
g(z)=\frac{-z p}{(z-p)(1-p z)}=w_{0}+\frac{p w_{0}}{(z-p)(1-p z)}(1-z)^{2} \in \Sigma^{\mathrm{s}}\left(p, w_{0}\right)
$$

where $w_{0}=-p /(1-p)^{2}$.

Here we also note that

$$
\operatorname{Re}\left(\frac{(z-p)(1-z p) g^{\prime}(z)}{f(z)+\frac{p}{(1-p)^{2}}}\right)=-(1-p)^{2} \operatorname{Re}\left(\frac{1+z}{1-z}\right)<0
$$

for all $z \in \mathbb{D}$ and $g$ satisfies the normalization condition $g(0)=0=g^{\prime}(0)-1$ whenever $w_{0}=-p /(1-p)^{2}$.

REMARK. In view of the last theorem, the corollary that follows from Theorem 9 in [6] is also not true since it uses the incorrect estimate for $\left|a_{-1}\right|$.

Acknowledgements. The authors thank Prof. K.-J. Wirths for his suggestions on various stages of work on this paper and for his continuous encouragement.

\section{References}

[1] F. G. Avkhadiev, Ch. Pommerenke and K.-J. Wirths, On the coefficients of concave univalent functions, Math. Nachr. 271 (2004), 3-9.

[2] F. G. Avkhadiev and K.-J. Wirths, Convex holes produce lower bounds for coefficients, Complex Variables 47 (2002), 553-563.

[3] - - -, On a conjecture of Livingston, Mathematica (Cluj) 46 (69) (2004), 19-23. 
[4] F. G. Avkhadiev and K.-J. Wirths, A proof of Livingston conjecture, Forum Math. 19 (2007), 149-158.

[5] B. Bhowmik, S. Ponnusamy and K.-J. Wirths, Domains of variability of Laurent coefficients and the convex hull for the family of concave univalent functions, Kodai Math. J. 30 (2007), 385-393.

[6] A. E. Livingston, Convex meromorphic mappings, Ann. Polon. Math. 59 (1994), $275-291$.

[7] J. Miller, Convex and starlike meromorphic functions, Proc. Amer. Math. Soc. 80 (1980), 607-613.

[8] J. Pfaltzgraff and B. Pinchuk, A variational method for classes of meromorphic functions, J. Anal. Math. 24 (1971), 101-150.

[9] St. Ruscheweyh, Two remarks on bounded analytic functions, Serdica 11 (1985), 200-202.

[10] K.-J. Wirths, On the residuum of concave univalent functions, Serdica Math. J. 32 (2006), 209-214.

[11] Y. L. Zhang [= Chang Yuh Lin], On the representation formulas for the functions in class $\Sigma^{*}\left(p, w_{0}\right)$, Proc. Amer. Math. Soc. 103 (1988), 517-520.

[12] Y. L. Zhang and S. Owa, Some remarks on a class of meromorphic starlike functions, Indian J. Pure Appl. Math. 21 (1990), 833-840.

Department of Mathematics

Indian Institute of Technology Madras

Chennai 600 036, India

E-mail: ditya@iitm.ac.in samy@iitm.ac.in 